\title{
Simulating the Need for Hospital Care for Circulatory Diseases
}

\author{
S.N. Cherkasov ${ }^{1,2}$, N.V. Savvina, ${ }^{1, *}$, V.S. Oleynikova ${ }^{2}$, A.V. Fedyaeva ${ }^{2}$ and I.L.
}

Savvina $^{1}$

${ }^{1}$ M.K. Ammosov North-Eastern Federal University, Yakutsk, Russia

${ }^{2}$ V.A. Trapeznikov Institute of Management Problems of Russian Academy of Sciences, Moscow, Russia

*Corresponding author. Email: nadvsavvina@mail.ru

\begin{abstract}
Inpatient care requires far more resources than outpatient care. In this regard, prognosing its needs is a more important component of the resource management of health organizations and the health system as a whole. It is relevant to create demand models for strategic regional planning. The purpose of this article is to present simulating inpatient care need for diseases of the circulatory system, taking into account gender characteristics. The purpose of the research is simulating inpatient care need for circulatory diseases, taking into account gender characteristics. Data of 420000 patients were used (by 210000 males and females). The analysis was carried out in relation to the days of inpatient treatment in 6 five-year old age groups: 31-35 years old, 36-40 years old, 41-45 years old, 46-50 years old, 51-55 years old, 56-60 years old. Regression analysis was performed to simulate age dynamics of need. The degree of predictor variables influence was evaluated by calculating the residual variance. A decrease in the level of hospital care need in men in older age groups is specific to diseases characterized by increased blood pressure (I10 - I15), while in relation to the causes associated with cerebrovascular diseases (I60 - I69) and coronary heart diseases (I20 - I25), the age dynamics of need is described by increasing power functions. In women, the dynamics of demanded volumes in medical care provided in inpatient conditions, in cerebrovascular diseases (I60 - I69), diseases characterized by increased blood pressure (I10 - I15) and coronary heart diseases (I20 - I25) is characterized by an upward trend throughout the studied age period, which can be described using power function. The mathematical analysis confirmed the paramount importance of age in hospital care need provided in inpatient conditions for circulatory diseases.
\end{abstract}

Keywords: hospital care, circulatory diseases, simulating inpatient care

\section{INTRODUCTION}

Increasing the impact of the health care system is an urgent task that can be solved with a systematic approach and adequate planning of the medical organizations work $[1,2]$. One promising way to improve the healthcare planning system is to use data in the planning process to analyze the dependence on the frequency of medical care episodes, that is, its demanded volumes, on age and gender. The knowledge of the required resources avoids "resource crises" and knowledge of the age periods when the need increases, plans preventive measures aimed at reducing the consumption level of inpatient medical care. The demand model developing this approach allows calculations to be made using data on the age-gender structure of the population. In the second stage, you can also use data on incidence levels [3-6].

Inpatient care requires far more resources than outpatient care. In this regard, forecasting its needs is a more important component of the resource management of health organizations and the health system as a whole. It is relevant to create the demand models for strategic regional planning [7-9].

\section{MATERIALS AND METHODS}

\subsection{Materials}

The International Classification of Diseases of the $10^{\text {th }}$ edition (ICD-10) was used as the basic 
classification of diseases. The name of the Class is given in accordance with the ICD edition at the time of the research. 420,000 inpatient hospitalization data were used (210,000 males and 210,000 females) per one year [3]. The cause for treatment or hospitalization was recorded in the primary medical records and entered a single database. The analysis included days of inpatient treatment depending on age in six five-year age groups: 31-35 years old, 36-40 years old, 41-45 years old, 46-50 years old, 51-55 years old, 56-60 years old [4].

The causes for hospitalization were ranked by Groups A, B, and C. The division into three Groups A, $\mathrm{B}$, and $\mathrm{C}$ suggests a differentiated approach to planning the health care need. For example, the causes included in Group A almost completely determine the magnitude of the total need for medical care and even the minor changes associated with this group of causes immediately affect the amount of need. It is particularly important to take into account the age-gender changes in the structure of causes and, above all, the changes occurring with respect to the causes included in Group A [5]. The optimal number of causes to be included in Group A is $2-3$. This article presents only the results of simulating the causes of hospitalizations in the Cause Group A.

\subsection{Data processing methods}

Analysis of age dynamics was carried out by classical methods of constructing statistical series. To identify the defining trends, the statistical series were smoothed by the three-point moving average method. During the analysis, the basic indicators of the statistical series were calculated: absolute increase, relative increase, visibility indicator and growth rate of the indicator.

Mathematical analysis using least squares algebraic functions (regression analysis) was conducted to simulate the age dynamics of the need for medical care. In the first step of the regression analysis, the obtained data were tried to be reproduced by a linear model. The quality of the created models was evaluated by $\mathrm{R}^{2}$ criterion (approximation coefficient). For the purposes of this study, the minimum value of the approximation factor should have been 0.75 .

While obtaining data of linear model inadequacy, that is, its inability to qualitatively reflect the observed dynamics, a second step was made, which was to add one or more non-linear terms in order to clarify the nature of the interaction between predictor variables.

The method used to estimate the parameters of the obtained regression was nonlinear estimation as a generalization of multiple regression and dispersion analysis, taking into account that the multiple regression method (and in dispersion analysis) assumes that the dependence of the response from predictor variables is linear.

Formally, nonlinear estimation is a universal approximating procedure evaluating any kind of dependence between a response variable and a set of independent variables. In general, all regression models created can be written in the form of the formula:

$\mathrm{y}=\mathrm{F}\left(\mathrm{x}_{1}, \mathrm{x}_{2}, \ldots . \mathrm{x}_{\mathrm{n}}\right)$

When conducting regression, and in particular, nonlinear regression analysis, we were interested in whether and if so, how, a dependent variable and a set of independent variables are related. The expression $\mathrm{F}(\mathrm{x} . .$.$) in the above expression means that the response$ variable $\mathrm{y}$ is a function of the independent variable $\mathrm{x}$.

As a result of this approach, a system of equations, linear and non-linear, was obtained, in each of which the dependence of the volume of medical care on predictor variables was expressed. In this case, age and gender acted as predictor variables. Each group of states (nosologies) corresponded to one or more equations. Several equations were used if different dynamics were observed at different age intervals. Then, an age interval was prescribed for each equation, at which exactly this regression equation should be used.

Finally, all the resulting equations were reduced to a single system, which made it possible to assess the total amount of hospital care need.

$Y_{1}=F_{1}\left(x_{1}, x_{2}, \ldots x_{n}\right)$
$Y_{2}=F_{2}\left(x_{1}, x_{2}, \ldots . x_{n}\right)$
$Y_{3}=F_{3}\left(x_{1}, x_{2}, \ldots . x_{n}\right)$
$Y_{4}=F_{4}\left(x_{1}, x_{2}, \ldots . x_{n}\right)$
$\ldots \ldots \ldots . . . \ldots . \ldots . . . .$.
$Y_{n}=F_{n}\left(x_{1}, x_{2}, \ldots x_{n}\right)$

Where $\mathrm{Yi}$ is the amount of necessary medical care for i pathological condition (nosology) and a separate type of medical care; Fi is a function. Determined by the study; xi are predictor variables.

The degree of influence of the studying variable age was estimated by calculating the residual dispersion as the sum of squares of deviations of the theoretical value of the volume of medical care need from the actual observed one. The formula for calculating the residual dispersion is shown below.

$D_{\text {residual dispersion }}=\sum_{1}^{n} \frac{\varepsilon^{2}}{n-2}$

where $\varepsilon-$ the square of the difference between the real demand value (dependent variable) and theoretically calculated using the regression equation. The sigmal deviation value was calculated as the square root from the dispersion.

$\sigma=\sqrt{D_{r d}}$

The degree of influence of age on the dependent variable (the magnitude of the care need) was defined as an empirical correlation by the formula: 


$$
\text { correlation ratio }=\sqrt{\frac{\left(D_{\text {total dispersion }}-D_{r d}\right)}{D_{t d}}}
$$

The total dispersion value of $D_{t d}$ was calculated by the standard method.

If the correlation ratio value was higher than 0.7 , then such a dependence was considered significant and the dependence of the demand value on the age of the patient was high. If the correlation ratio value was more than 0.9, the change in the value of the medical care need was almost completely determined by the patient's age. The difference between unit and correlation value was regarded as a proportion of the effect on the magnitude of the care need of other unaccounted factors.

\section{RESULTS OF THE RESEARCH}

\subsection{Results of need simulations in males.}

The largest amounts of inpatient care in males (first ranked) were for diseases characterized by high blood pressure (I10 - I15). The need dynamics was characterized by an increase level to the age of 55 years old. Growth rates were maximum in the 31-35 age group (29.5\% of annual demand growth) and minimum in the 51-55 age group (3.7 \% of annual demand growth). In the age group of 56-60 years old, there was a decrease in the level by $0.8 \%$ per year. Differences in the level of need in the age groups of 31-35 years old and 56-60 years old were 6.2 times.

Since there is a high probability of a further decrease in the need level with increasing age of a male, it is advisable to use a second-order parabola with a negative first coefficient as a modeling function. According to this model, the bed-day need can be expressed by the following formula:
$\mathrm{V}_{\text {inpatient (bed-day) }}=-19,73 \mathrm{x}^{2}+277,94 \mathrm{x}-158,02\left(\mathrm{R}^{2}=0,974\right)(4)$ (hereinafter $\mathrm{x}$ is the sequence number of the age interval)

The second ranking in terms of the sought-after inpatient medical care is occupied by a block of causes associated with cerebrovascular diseases (I60 - I69). The dynamics of inpatient care demand in males is characterized by an increase need throughout the studied age period. The highest growth rates are recorded in young age groups (37.9\% of annual demand growth), but despite the slowdown in growth, the difference between the age groups of 31-35 and 56-60 years old is 19 times, which is the highest for men. The levels of need achieved 1938 beds per 1000 of males of appropriate age seeking inpatient care are also maximum among the other blocks of causes of hospitalization. The described dynamics are modeled using a power function.

$\mathrm{V}_{\text {inpatient (bed-day) }}=93,005 \mathrm{x}^{1,672}\left(\mathrm{R}^{2}=0,992\right)$

The third rank in terms of the sought-after inpatient medical care is occupied by a block of causes associated with coronary heart diseases (I20 - I25). Demand dynamics are characterized by an increase in demand throughout the study age period. Unlike the previous blocks of causes, the highest growth rates are recorded in the age groups of 36-45 years old (27.6 and $19.4 \%$ of the annual increase in demand). In older age groups, the rate of growth in inpatient care needs decreased, but the difference between the age groups of 31-35 and 5660 is 17 times, and the level of demand achieved is 1,467 beds-days per 1,000 men of appropriate age seeking inpatient care. The described dynamics are modeled using a power function.

$\mathrm{V}_{\text {inpatient (bed-day) }}=68,204 \mathrm{x}^{1,68} \quad\left(\mathrm{R}^{2}=0,972\right)$

Characteristics of mathematical models of intensity dynamics of medical care consumption provided in inpatient conditions in males are presented in Table 1.

Table 1. Characteristics of mathematical models of intensity dynamics of medical care consumption (duration of inpatient treatment) provided in inpatient conditions in males (duration of inpatient treatment in bed days)

\begin{tabular}{|l|l|l|l|l|l|l|l|}
\hline Modeling state & Formula & $\sum \varepsilon^{2}$ & $D_{\text {ост }}$ & $\sigma_{\text {ост }}$ & $D_{y}$ & $\begin{array}{l}\text { The share of } \\
\text { influence of } \\
\text { the test feature } \\
\text { (age) }\end{array}$ & $\begin{array}{l}\text { The share of } \\
\text { random } \\
\text { fluctuations of the } \\
\text { resulting quantity }\end{array}$ \\
\hline block I10 - I15 & $Y=-19.73 x^{2}+277.94 x-158.02$ & 9501.0 & 1900.2 & 43.59 & 73244.9 & 0.986 & 0.014 \\
\hline block $160-169$ & $Y=93.005 x^{1.672}$ & 24534.8 & 4906.98 & 70.04 & 513569.8 & 0.995 & 0.005 \\
\hline block I20 - I25 & $Y=68.204 x^{1.68}$ & 26668.3 & 5333.7 & 73.03 & 306717.9 & 0.991 & 0.009 \\
\hline
\end{tabular}

\subsection{Results of need simulations in females}

The largest amounts of inpatient care in women, as well as in men (first ranked), were in demand for diseases characterized by high blood pressure (I10 I15). The dynamics are characterized by an increase level of need throughout the study age period of a woman's life. The largest increases are recorded in younger age groups. The annual growth rate of demand in the age group of 36-40 years old was $48.8 \%$. Such high rates are due to the "low base" of initial values, which suggests that it is young age that is critical for 
starting preventive measures of this type of pathology in women. In older age groups of women, the rate of increase in demand has gradually decreased to a minimum of $3.3 \%$ in the 56-60 age group. Differences in the level of need in the age groups of 31-35 years old and 56-60 years old are 22.2 times.

The described dynamics are modeled using a power function.

$\mathrm{V}_{\text {inpatient (bed-day) }}=40,248 \mathrm{x}^{1,868} \quad\left(\mathrm{R}^{2}=0,996\right)$

The dynamics of hospitalization cases of females for cerebral vessel diseases (I60 - I69) was also characterized by an upward trend throughout the age period. The maximum rate is recorded in the young age group (31-35 years old) and accounts $59 \%$ of the annual increase in demand. In older age groups of women, the growth rate decreases to a minimum $8.8 \%$ of the annual increase over the age of 50. During the 30year age period, the demand for outpatient visits has increased 39 times.

The described dynamics are modeled using a power function.
$\mathrm{V}_{\text {inpatient (bed-day) }}=45,914 \mathrm{x}^{2,1} \quad\left(\mathrm{R}^{2}=0,997\right)$

The largest gradient of growth in inpatient care need was recorded for coronary heart diseases (block I20 I25) as a cause for hospitalization. During the studied age period, the volume of sought-after medical care increased 140 times, which is significantly more than in other analyzed conditions. The greatest growth rate of demand was observed in the age group of 40-45 years old, after which the level of growth stabilized, but remained very significant. It is advisable to consider the age of 35 years old as a critical age. It should be noted that the highest value of the degree indicator among the models of all other causes for hospitalization is 2.84 . This determines a significant increase in demand with an increase in the age of the woman.

The described dynamics are also modeled using a power function.

$\mathrm{V}_{\text {inpatient (bed-day) }}=2,554 \mathrm{x}^{2,865} \quad\left(\mathrm{R}^{2}=0,98\right)$

Summary characteristics of mathematical models of intensity dynamics of medical care consumption provided in inpatient conditions in females are presented in Table 2.

Table 2. Characteristics of mathematical models of the dynamics of the intensity of consumption of medical care provided in inpatient conditions in females for diseases characterized by high blood pressure (block I10 - I15)

\begin{tabular}{|l|l|l|l|l|l|l|l|}
\hline Modeling state Formula & $\sum \varepsilon^{2}$ & $D_{\text {ост }}$ & $\sigma_{\text {ост }}$ & $D_{y}$ & $\begin{array}{l}\text { The share of influence } \\
\text { of the test feature (age) }\end{array}$ & $\begin{array}{l}\text { The share of random } \\
\text { fluctuations of the resulting } \\
\text { quantity }\end{array}$ \\
\hline block $110-115$ & $\mathrm{Y}=40.248 \mathrm{x}^{1.868}$ & 2437.8 & 4947.5 & 70.3 & 151409.7 & 0.984 & 0.016 \\
\hline block $160-169$ & $\mathrm{Y}=45.914 \mathrm{x}^{2.1}$ & 2821.6 & 564.3 & 23.8 & 537920.5 & 0.999 & 0.001 \\
\hline block $120-125$ & $\mathrm{Y}=2.554 \mathrm{x}^{2.865}$ & 64.5 & 12.9 & 3.59 & 410.5 & 0.984 & 0.016 \\
\hline
\end{tabular}

\section{CONCLUSION}

The analysis of the age-related dynamics of inpatient care needs to conditions determining the baseline of need showed that, regardless of cause and gender, the volume of inpatient care provided recorded an increasing trend to the volume of need. However, analysis of individual cause groups allows forming a differentiated approach to planning. In males, one of three cause groups defining the baseline demand value can be simulated using second-order parabolas with a negative first coefficient. This choice of function is due to lower requirements for older age groups. This is typical for diseases characterized by high blood pressure (I10 - I15). With regard to the causes of cerebrovascular diseases (I60 - I69) and coronary heart diseases (I20 I25), the age dynamics of need is described by power functions, which is determined by the constant increase in need throughout the study period.

In females, the baseline need for inpatient care is also determined by three groups of causes. The dynamics of demanded volumes in medical care provided in stationary conditions, in cerebrovascular diseases [cerebral vessel disease] (I60 - I69), diseases characterized by high blood pressure (I10 - I15) and coronary heart diseases (I20 I25) is characterized by an upward trend throughout the studied age period, which can be described by power function. For all these conditions, the use of the same functions and similarity of the values of the degree indicator in the simulating process confirms the presence of common mechanisms and factors underlying the need for outpatient and inpatient medical care.

The mathematical analysis confirmed the paramount importance of age for medical care need provided in inpatient conditions for diseases of the circulatory system. The revealed patterns should be taken into account when planning the necessary amounts of inpatient medical care, including the age-gender characteristics of the territory where planning is carried out.

\section{REFERENCES}

[1] R.U. Khabriev, A.L. Lindendraten, Yu.M. Komarov, The strategy of health care of population as a background of public social policy, Probl. of 
soc. hyg., health care and the hist. of med. 3 (2014) 3-5.

[2] U. Genovese, S. Del Sordo, M. Casali et al., A new paradigm on health care accountability to improve the quality of the system: four parameters to achieve individual and collective accountability, J. of Glob. Health 7(1) (2017) 010301. Retrieved from: https://doi.org/ 10.7189/jogh.07.010301

[3] R.G. Oganov, G.Ya. Maslennikova, Demographic trends in the Russian Federation: the contribution of diseases of the circulatory system, Cardiovasc. therapy and prevent. 11(1) (2012) 5-10.

[4] E.V. Bolotova, I.V. Samorodskaya, I.M. Komissarova, Gender and age characteristics of the prevalence of risk factors for cardiovascular diseases in the rural population of the Krasnodar Territory, Cardiovasc. therapy and prevent. 14(1) (2015) 47-52.

[5] A. Saeed, J. Kampangkaew, V. Nambi, Prevention of Cardiovascular Disease in Women, Method. Debakey Cardiovasc. J. 13(4) (2017) 185-192.

[6] R. Basu, L. Franzini, P.M. Krueger, D.R. Lairson, Gender disparities in medical expenditures attributable to hypertension in the United States, Wom. Health Issues 20(2) (2010) 114-25.

[7] K.G.M. Moons, A.P. Kengne, D.E. Grobbee et al., Risk prediction models: II. External validation, model updating, and impact assessment, Heart. 98(9) (2012) 691-698.

[8] P. Goyal, T. Paul, Z.I. Almarzooq, J.C. Peterson et al., Sex- and Race-Related Differences in Characteristics and Outcomes of Hospitalizations for Heart Failure With Preserved Ejection Fraction, J. Am. Heart Assoc. 29(6(4)) (2017) e003330.

[9] R.A. Nishimura, J.A. Linderbaum, J.M. Naessens et al., A nonresident cardiovascular inpatient service improves residents' experiences in an academic medical center: a new model to meet the challenges of the new millennium, Acad. Med. 79(5) (2004) 426-431. 